\title{
Considerando Estilos de Aprendizagem, Emoções e Personalidade em Informática na Educação
}

\author{
Considering Learning Styles, Emotions and \\ Personality in Computers in Education
}

\author{
Janderson Jason Barbosa Aguiar \\ Universidade Federal de Campina Grande
}

\begin{abstract}
Resumo: Muitas vezes tratam-se turmas homogeneamente e as TDIC (Tecnologias Digitais de Informação e Comunicação) são utilizadas pelos alunos desconsiderando que eles possuem particularidades (tais como: estilos de aprendizagem, emoções e traços de personalidade) que influenciam em sua aquisição de conhecimento. Neste trabalho, são apresentados conceitos e reflexões visando incentivar discussões e estudos teóricos ou empíricos que considerem essas particularidade dos alunos durante o processo de ensino e aprendizagem. É apresentada também uma breve análise de dados de alunos de computação, em diferentes níveis de ensino, os quais responderam a questionários visando identificar perfis referentes a seus estilos de aprendizagem e a seus traços de personalidade. A análise corrobora com a ideia da heterogeneidade de perfis, mesmo considerando alunos da mesma área/nível de ensino. Percebe-se que ainda há várias possibilidades de pesquisas sobre aliar as TDIC e as particularidades de alunos objetivando a melhoria do processo de ensino e aprendizagem.
\end{abstract}

Palavras-chave: Informática na educação. Estilos de aprendizagem. Emoções. Personalidade. Computação afetiva.

\begin{abstract}
It is common to treat classes homogenously and use DICT (Digital Information and Communication Technologies) disregarding that students have special features (such as learning styles, emotions and personality traits) that influence in their acquisition of knowledge. This paper presents concepts and reflections to encourage discussion and theoretical or empirical research on student's peculiarities during the teaching and learning process. This paper also presents a brief analysis of student data (computing students at different levels of education). These students answered questionnaires to identify profiles related to their learning styles and personality traits. The analysis supports the idea that there is heterogeneity of student profiles, even students from the same area/level of education. One can see that there are several possibilities for research on combining the DICT and particularities of students aiming at the improvement of the teaching and learning process.
\end{abstract}

Keywords: Computers in education. Learning styles. Emotions. Personality. Affective computing. 


\section{I ntrodução}

As Tecnologias Digitais de Informação e Comunicação (TDIC) são constantemente utilizadas no processo educativo como meio facilitador do processo de ensino e aprendizagem (COSTA, AGUIAR e MAGALHÃES, 2013). Uma vez que essas TDIC fazem parte do cotidiano de muitos alunos, especialmente em cursos de área tecnológica, é interessante intensificar os estudos sobre como usar essas tecnologias na educação, de forma a prover cada vez mais estratégias motivadoras, tais como a personalização do ambiente, a fim de maior desempenho por parte dos alunos.

Independente da área do curso, na modalidade a distância, é necessário que os alunos interajam constantemente com as TDIC. Em relação à modalidade presencial, apesar de haver cursos (especialmente em áreas não tecnológicas) que não fazem uso constante dessas tecnologias, há também cursos (principalmente na área tecnológica), a exemplo dos cursos de computação, nos quais os alunos são frequentemente submetidos à interação com as TDIC, além de geralmente apresentarem maior facilidade de manejo (sendo, portanto, relevante e de fácil aceitação/adaptação, por parte de tais alunos, usar as TDIC para viabilizar meios para facilitar sua aprendizagem).

Ao comentar sobre alunos de área tecnológica, é importante destacar que ainda é comum ouvir comentários sobre estereótipos, considerando homogeneamente os aprendizes dessa área. Entretanto, observa-se que, embora estejam na mesma área, ou até no mesmo curso, esses indivíduos apresentam particularidades - e essas particularidades podem influenciar veementemente no processo de ensino e aprendizagem.

Dentre as particularidades a considerar, é possível citar conceitos como Estilos de Aprendizagem, Emoções e Traços de Personalidade. Segundo Valaski, Malucelli e Reinehr (2011), a identificação dos estilos de aprendizagem dos alunos é uma das formas para a obtenção de suas preferências, propiciando materiais de aprendizagem mais efetivos no processo de ensino e aprendizagem. Segundo Nunes (2012), os aspectos psicológicos, tais como emoções e personalidade, têm importância e influenciam o processo de tomada de decisão dos humanos.

Jaques e Nunes (2012) afirmam que a Computação Afetiva - área de pesquisa interessada em investigar como os computadores podem inferir e expressar emoções e personalidade (JAQUES et al., 2012) - pode contribuir na formação da presença social dos alunos em Ambientes Virtuais de Aprendizagem (AVA), tendo em vista que os seres humanos vivem em grupos de pessoas com características similares e/ou complementares às suas. Além disso, Jaques e Nunes (2012) defendem que inferir estados afetivos do aluno pode auxiliar a prever aspectos como evasão e baixo desempenho.

A adoção de aspectos psicológicos humanos na educação mediada por TDIC não está consolidada. Como é exposto por Nunes (2012), ainda há dificuldade em extrair intencionalmente esses aspectos. Entretanto, há pesquisas emergentes que vêm demonstrando a importância crescente desses aspectos, como as que são citadas ao longo deste artigo. 
A partir do exposto, percebe-se que conceitos como Estilos de Aprendizagem, Emoção e Personalidade são pertinentes para se considerar em Informática na Educação, especialmente no sentido de personalização de ambientes educacionais.

Neste trabalho, são apresentados conceitos e reflexões visando incentivar estudos teóricos ou empíricos que considerem empregar esses conceitos durante o processo de ensino e aprendizagem. Na Seção 2, são apresentados alguns trabalhos relacionados. Os fundamentos sobre Estilos de Aprendizagem, Emoções e Personalidade são detalhados, respectivamente, nas Seções 3, 4 e 5. Por fim, na Seção 6 são realizadas algumas considerações.

Ressalta-se que, uma vez que os Estilos de Aprendizagem e a Personalidade são características mais estáveis nos indivíduos (bem menos mutáveis que, por exemplo, Emoções), nas Seções 3 e 5 são também apresentados resultados de uma breve análise de dados empíricos, a fim de demonstrar que, mesmo alunos de um mesmo curso e do mesmo nível de ensino (nível técnico, graduação, mestrado, doutorado), possuem características diferentes relativas a estilos de aprendizagem e traços de personalidade.

\section{Trabalhos Relacionados}

Uma vez que este artigo apresenta aspectos e discussões a fim de fomentar o debate acadêmico de linhas de pesquisa envolvendo Estilos de Aprendizagem, Emoções e Personalidade em Informática na Educação - e, portanto, proporcionar reflexões para direcionamento teórico/empírico - é possível encontrar trabalhos similares que abordem reflexões sobre esses conceitos.

Na pesquisa de Silva et al. (2013), ao serem investigados cursos de especialização para identificar se os estilos de aprendizagem impactam o desempenho acadêmico nas atividades de avaliação on-line e presencial na Educação a Distância (EAD), são realizados comentários e apresentados conceitos importantes relativos a estilos de aprendizagem. Aguiar, Fechine e Costa (2014) apresentaram um estudo mapeando as iniciativas brasileiras em Informática na Educação que utilizaram os conceitos de Estilos Cognitivos e Estilos de Aprendizagem.

Özyurt e Özyurt (2015) realizaram uma análise do conteúdo de trabalhos recentes (entre 2005 e 2014) sobre hipermídia educacional adaptativa com base em estilos de aprendizagem, discutindo os potenciais tópicos de investigação, e analisando as tendências e as lacunas existentes na literatura. Nesse contexto, Truong (2016) também realizou uma revisão na literatura sobre como estilos de aprendizagem estão sendo integrados em sistemas e-learning adaptativos, fornecendo recomendações e orientações para pesquisas futuras.

Nunes et al. (2010) apresentaram aspectos relacionados à Computação Afetiva e sua influência na personalização de ambientes educacionais, com enfoque na geração de equipes compatíveis em AVA na EAD. Na pesquisa de Nunes (2012), são apresentadas pesquisas científicas e tecnológicas realizadas no âmbito da Computação Afetiva relacionada à personalização de interfaces, interações e recomendações de produtos, serviços e pessoas em ambientes computacionais. No documento elaborado como base de um minicurso ministrado por Jaques e Nunes (2012), é provida uma visão geral teórica e prática relativa à Computação 
Afetiva aplicada em ambientes computacionais de aprendizagem. No trabalho de Jaques et al. (2012), além de ser abordada a Computação Afetiva, foi realizada uma associação com Sistemas Tutores Inteligentes, apresentando desafios e perspectivas de investigações futuras nessas áreas.

Longhi (2011), ao abordar vários conceitos importantes sobre aspectos afetivos, confirmou a hipótese de que os estados de ânimo de um aluno podem ser reconhecidos em um AVA por meio de um modelo computacional que inter-relacione fatores de personalidade $e$ comportamento. Komulainen et al. (2014) realizaram um estudo objetivando apresentar em que as características de personalidade representam na vida diária, investigando o efeito dos traços de personalidade sobre diferentes processos emocionais diários, utilizando, para isso, informações de estudantes universitários. Faquin, Falci e Araújo (2016) realizaram considerações relativas à personalidade ao apresentar os resultados de seu trabalho, cujo objetivo foi compreender como perfis de personalidade distintos podem se relacionar a diferentes desempenhos acadêmicos e níveis de habilidade em alunos de Sistemas de Informação.

Ao indicar resultados revelando que agentes pedagógicos animados têm impactos diferentes no comportamento do aluno segundo a sua personalidade, Nunes e J aques (2014) realizaram, em seu trabalho, considerações relativas a emoções e personalidade. Reis et al. (2015) publicaram um trabalho relativo ao estado da arte sobre afetividade na formação de grupos em ambientes colaborativos de aprendizagem; dentre os resultados, foi indicado que a maioria dos estudos selecionados considerou os traços de personalidade na formação de grupos em ambientes de aprendizagem colaborativa com suporte computacional.

Li e Armstrong (2015) investigaram a relação entre estilos de aprendizagem e personalidade em gestores internacionais. No contexto de examinar como emoções de adultos afetam em seus estilos de aprendizagem, Samsudin e Ch'ng (2015) verificaram que esses dois conceitos estão inter-relacionados e afetam as atividades de aprendizagem diárias.

Destaca-se que, além desses trabalhos, é possível encontrar vários outros que, apesar de não necessariamente realizarem reflexões enfatizando os conceitos de Estilos de Aprendizagem, Emoções e/ou Personalidade, usaram pelo menos um desses conceitos como parte de sua pesquisa. Para isso sugere-se que, assim como realizado no desenvolvimento deste trabalho, sejam realizadas buscas por artigos recentes via Portal de Periódicos CAPES/MEC (vislumbrando pesquisas em âmbito internacional), além de realizar buscas de tais conceitos nos seguintes veículos brasileiros de publicação científica: Revista Informática na Educação: Teoria \& Prática (IETP), Revista Brasileira de Informática na Educação (RBIE), Revista Novas Tecnologias na Educação (RENOTE), Anais do Workshop de Informática na Escola (WIE), Anais do Simpósio Brasileiro de Informática na Educação (SBIE).

No Quadro 1, em síntese, estão listados os trabalhos citados nesta seção, sendo indicados quais trabalhos estão relacionados com quais conceitos. $O$ símbolo $\checkmark$ indica que determinado trabalho apresentou considerações relacionadas a determinado conceito; por outro lado, o 
símbolo X indica que determinado trabalho não está intrinsecamente relacionado a determinado conceito.

Quadro 1 - Comparação entre trabalhos relacionados.

\begin{tabular}{|c|c|c|c|}
\hline Trabalho & Estilos de Aprendizagem & Emoções & Personalidade \\
\hline Aguiar, Fechine e Costa (2014) & $\checkmark$ & $X$ & $X$ \\
\hline Faquin, Falci e Araújo (2016) & $X$ & $X$ & $\checkmark$ \\
\hline Jaques e Nunes (2012) & $X$ & $\checkmark$ & $\checkmark$ \\
\hline Jaques et al. (2012) & $X$ & $\checkmark$ & $\checkmark$ \\
\hline Komulainen et al. (2014) & $X$ & $\checkmark$ & $\checkmark$ \\
\hline Li e Armstrong (2015) & $\checkmark$ & $\checkmark$ & $\checkmark$ \\
\hline Longhi (2011) & $X$ & $\checkmark$ & $\checkmark$ \\
\hline Nunes (2012) & $X$ & $\checkmark$ & $\checkmark$ \\
\hline Nunes et al. (2010) & $X$ & $\checkmark$ & $\checkmark$ \\
\hline Nunes e Jaques (2014) & X & $\checkmark$ & $\checkmark$ \\
\hline Özyurt e Özyurt (2015) & $\checkmark$ & $\checkmark$ & $\checkmark$ \\
\hline Reis et al. (2015) & $\checkmark$ & $X$ & $X$ \\
\hline Samsudin e Ch'ng (2015) & $\checkmark$ & $X$ & $X$ \\
\hline Silva et al. (2013) & $\checkmark$ & $X$ \\
\hline Truong (2016) & & & \\
\hline
\end{tabular}

Fonte: elaborado pelo autor.

A diferença básica desses trabalhos, em relação a este, consiste principalmente no fato que, neste artigo, são reunidos os três conceitos (Estilos de Aprendizagem, Emoções e Personalidade), sendo referenciados vários trabalhos importantes que podem servir de base para os leitores. Além disso, este trabalho difere de outros citados por realizar uma breve análise de dados de alunos de computação em diferentes níveis de ensino (referentes a seus Estilos de Aprendizagem e seus Traços de Personalidade), sendo também comentadas algumas perspectivas de uso dos conceitos de Estilos de Aprendizagem, Emoções e Personalidade na área de educação em computação.

\section{Estilos de Aprendizagem}

Embora não seja algo consensual na educação, há docentes e pesquisadores preocupados com as formas com que os aprendizes lidam particularmente com as informações, sendo o conceito de Estilos de Aprendizagem levado em consideração em muitas pesquisas atualmente.

No âmbito de Informática na Educação, muitos autores usam o termo Estilos Cognitivos como sinônimo de Estilos de Aprendizagem, e outros ainda usam o termo Estilos Cognitivos de Aprendizagem, sendo sugerido, por Aguiar, Fechine e Costa (2014), o uso do termo "Estilos de Aprendizagem" ou "Estilos Cognitivos de Aprendizagem" quando o foco for relativo à aprendizagem e à aquisição de informação - enquanto "Estilos Cognitivos" foca no controle e na organização dos processos cognitivos.

Há vários modelos teóricos empregados em relação à forma como o aluno aprende (COFFIELD et al., 2004), sendo o de Felder e Silverman (1988) um dos mais populares. Ao 
adotar esse modelo, é comum a utilização do questionário ILS (Index of Learning Styles) para extração dos estilos de aprendizagem (FELDER e SOLOMAN, 1999). Esse questionário identifica estas quatro dimensões para formas de aprendizado: 1-Ativo/Reflexivo, 2-Sensorial/Intuitivo, 3-Visual/Verbal e 4-Sequencial/Global. No Quadro 2, são apresentadas características de ambas as categorias dessas quatro dimensões.

Quadro 2 - Características dos Aprendizes de acordo com seus Estilos de Aprendizagem.

\begin{tabular}{|c|c|}
\hline Dimensão & Características dos Aprendizes \\
\hline $\begin{array}{c}\text { Ativo } \\
\text { Reflexivo }\end{array}$ & $\begin{array}{l}\text { Ativos: tendem a reter e compreender melhor a informação participando ativamente de } \\
\text { alguma atividade, discutindo, aplicando ou explicando para outros; gostam do trabalho em } \\
\text { grupo; são rápidos, mas podem ser precipitados. } \\
\text { Reflexivos: preferem refletir calmamente sobre a informação; podem ser mais lentos para } \\
\text { iniciar uma atividade; gostam do trabalho individual ou em dupla. }\end{array}$ \\
\hline $\begin{array}{l}\text { Sensorial } \\
\text { Intuitivo }\end{array}$ & $\begin{array}{l}\text { Sensoriais: gostam de resolver problemas por meio de procedimentos bem estabelecidos e } \\
\text { não apreciam complicações e surpresas; são pacientes com detalhes; memorizam fatos com } \\
\text { facilidade; apreciam trabalhos de manipulação, experimentais, repetitivos (laboratório); } \\
\text { tendem a ser práticos e cuidadosos; não gostam de disciplinas que não têm uma conexão } \\
\text { aparente com o mundo real. } \\
\text { Intuitivos: frequentemente preferem descobrir possibilidades e inter-relações; gostam de } \\
\text { inovação e não apreciam a repetição; sentem-se confortáveis com abstrações e formulações } \\
\text { matemáticas; são rápidos e criativos; não apreciam as disciplinas que envolvem muita } \\
\text { memorização e cálculos rotineiros; apreciam a variedade. }\end{array}$ \\
\hline $\begin{array}{l}\text { Visual } \\
\text { Verbal }\end{array}$ & $\begin{array}{l}\text { Visuais: lembram-se mais do que viram; substituem as palavras por símbolos; preferem as } \\
\text { representações visuais - diagramas, quadros, cronogramas, gráficos, filmes e } \\
\text { demonstrações; reconstroem imagens de diferentes modos. } \\
\text { Verbais: tiram mais proveito das explanações escritas e faladas; gostam de ouvir e de } \\
\text { tomar notas; materiais de apoio impressos são úteis para eles; repetem as palavras, } \\
\text { falando ou escrevendo; fazem a leitura de suas notas em silêncio; transformam diagramas } \\
\text { em palavras. }\end{array}$ \\
\hline $\begin{array}{c}\text { Sequencial } \\
\text { Global }\end{array}$ & $\begin{array}{l}\text { Sequenciais: avançam com entendimento parcial; ganham entendimento em passos } \\
\text { lineares, com cada passo derivado do anterior; tendem a seguir caminhos lógicos e graduais } \\
\text { na solução de um problema; têm facilidade para explicar; enfatizam a análise, os detalhes. } \\
\text { Globais: aprendem em grandes saltos, absorvendo o material quase que aleatoriamente, } \\
\text { sem enxergar conexões, e repentinamente compreendem tudo; precisam do contexto, do } \\
\text { grande quadro; são hábeis para resolver rapidamente problemas complexos; têm facilidade } \\
\text { para juntar elementos, de maneiras novas, uma vez que tenham enxergado o grande } \\
\text { quadro, mas podem ter dificuldade para explicar como fizeram isso; o foco está na síntese, } \\
\text { no pensamento sistêmico, holístico. }\end{array}$ \\
\hline
\end{tabular}

Fonte: Silva et al. (2013), p. 4, adaptado de Felder e Silverman (1988).

Ressalta-se que há estudos que reforçam que o ILS é um instrumento confiável, válido e adequado para identificação dos estilos de aprendizagem (FELDER e SPURLIN, 2005; LITZINGER et al., 2007; ZYWNO, 2003). Todavia, nesses estudos é recomendado que continuem a ser realizadas pesquisas para avaliar a aplicação de tal instrumento.

Como exemplo, utilizando a teoria de Felder e Silverman (1988), a partir do questionário ILS, são ilustrados em gráficos, na Figura 1, alguns perfis de alunos de computação, em diferentes níveis, com base em seus estilos de aprendizagem.

Na Figura 1(a) são apresentados dois perfis bem diferentes de alunos em um curso técnico. Destaca-se o percentual alto para o estilo Reflexivo do Tecnolando B e, por outro lado, o baixo índice desse estilo para o Tecnolando A (ou seja, o Tecnolando A possui predominantemente o estilo Ativo). Outra diferença acentuada é o percentual alto para o estilo Verbal do Tecnolando 
A e, por outro lado, o baixo índice desse estilo para o Tecnolando B (ou seja, o Tecnolando B possui predominantemente o estilo Visual).

Na Figura 1(b), em relação a graduandos em computação, são vistos novamente dois perfis distintos. Destaca-se o percentual maior para o estilo Global do Graduando B em relação ao Graduando A (ou seja, o Graduando A possui predominantemente o estilo Sequencial).

Na Figura 1(c), é apresentada uma comparação similar considerando mestrandos em computação. Na Figura 1(d), perfis de doutorandos em computação.

Figura 1 - Exemplos de perfis de alunos de computação, com Estilos de Aprendizagem distintos, em diferentes níveis de ensino: (a) técnico; (b) graduação; (c) mestrado; (d) doutorado.

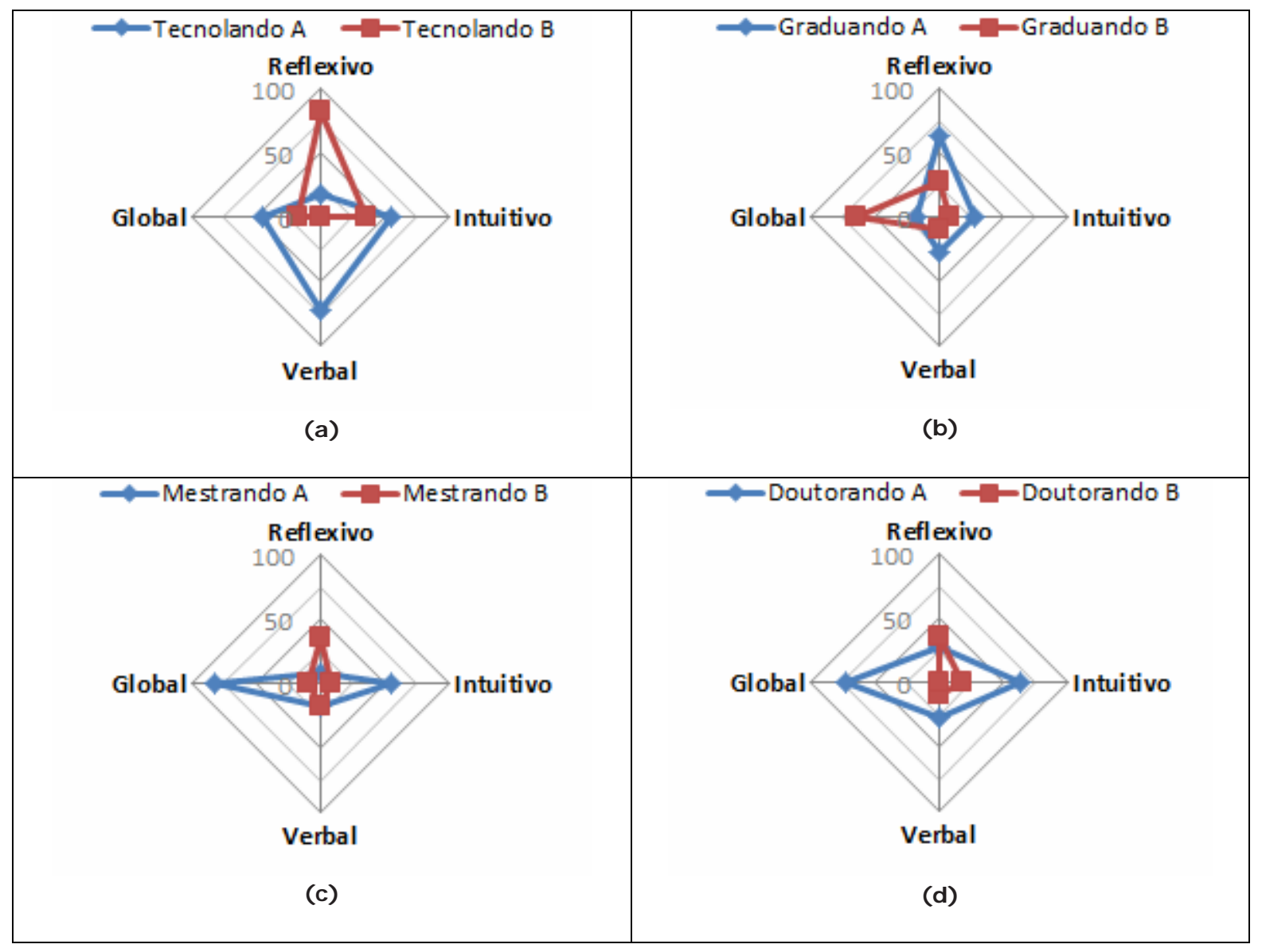

Fonte: elaborada pelo autor.

Os dados apresentados na Figura 1 foram obtidos com base na participação de 118 alunos de computação (61 alunos em nível técnico, 36 alunos de graduação e 21 pós-graduandos mestrandos ou doutorandos), os quais responderam às questões do ILS, via formulário on-line. A partir das respostas, foi definido, para caracterizar cada aluno, um vetor com 4 elementos (dados numéricos entre 0 a 100) indicando o percentual dos estilos de aprendizagem em cada uma das dimensões de Felder e Silverman (1988). Por exemplo: 0 para a dimensão 1Ativo/Reflexivo indicaria 0\% para o Estilo Reflexivo e, consequentemente, 100\% para o Estilo 
Ativo; 34 para a dimensão 2-Sensorial/Intuitivo indicaria 34\% para o Estilo Intuitivo e, consequentemente, 66\% para o Estilo Sensorial; 57 para a dimensão 3-Visual/Verbal indicaria $57 \%$ para o Estilo Verbal e, consequentemente, $43 \%$ para o Estilo Visual; e 86 para a dimensão 4-Sequencial/Global indicaria $86 \%$ para o Estilo Global e, consequentemente, $14 \%$ para o Estilo Sequencial. Uma vez que cada aluno foi representado por um vetor, foi utilizada, para a comparação entre esses vetores, a Medida de Similaridade dos Cossenos (quanto mais próximo de zero for essa medida, mais baixa é a similaridade); assim, foram identificados os pares de alunos menos similares, ilustrando-se na Figura 1 seus perfis.

Os gráficos da Figura 1 ilustram como é possível observar a ideia de heterogeneidade de perfil relativa aos estilos de aprendizagem, entre os alunos de computação, independente do nível de ensino. Ressalta-se que os gráficos não são apresentados a fim de delinear um perfil de estilos de aprendizagem para tais cursos, mas para destacar a diferença de perfis de alunos em uma mesma área/nível de ensino.

É possível citar também iniciativas de uso do modelo de Felder e Silverman (1988) que não utilizam o ILS, utilizando métodos para inferir os estilos baseando-se em analisar como o estudante se comporta (por exemplo, que tipos de atividades realiza) em sistemas de aprendizagem (DORÇA et al., 2013; GRAF, KINSHUK e LIU, 2008; POPESCU, TRIGANO e BADICA, 2007). Além da obtenção dos estilos, alguns pesquisadores apresentam a possibilidade de atualizações dinâmicas dos perfis relativos aos estilos de aprendizagem dos alunos (DORÇA et al., 2013; POPESCU, TRIGANO e BADICA, 2007).

\section{Emoções}

Segundo Reis et al. (2015), pesquisas em Informática na Educação usam as terminologias "estados afetivos" e "aspectos afetivos" para caracterizar o estado mental afetivo (emoção, estado de ânimo) e as características de personalidade de um aluno, nas diversas situações vivenciadas no processo de ensino e aprendizagem.

Em relação à afetividade, Longhi (2011) indica que há diferentes conceituações, com base em abordagens filosóficas e psicológicas, sendo o modelo de Scherer (2000) vantajoso ao evidenciar as diferenças entre emoções, estados de ânimo e traços de personalidade.

Scherer (2000) baseia-se em fatores como intensidade, duração, sincronização, impacto comportamental, entre outros, para diferenciar as emoções dos outros estados afetivos. Enquanto emoções são caracterizadas como estados afetivos de curta duração (minutos ou horas), alta intensidade e disparadas/ativadas por um estímulo (por exemplo: alegria, medo, raiva, tristeza e vergonha), os estados de ânimo (humor) caracterizam-se como estados afetivos difusos, de baixa intensidade, longa duração e sem causa aparente, e os traços de personalidade são definidos como estados afetivos que tendem a ser estáveis e característicos de um indivíduo (LONGHI, 2011; REIS et al., 2015).

As emoções podem ser classificadas em primárias/básicas (inatas e de segurança individual) e secundárias/sociais (estabelecidas a partir das primárias). Para mais detalhes sobre os 
conceitos dos diferentes estados afetivos, sugere-se a leitura da fundamentação teórica da tese de doutorado de Longhi (2011).

Gil et al. (2015) afirmam que aplicações para reconhecer emoções estão recebendo muita atenção como um meio para melhorar a experiência do usuário com a tecnologia. Esses autores indicam que há proliferação de projetos envolvendo a coleta de dados de usuários relativos a emoções, como, por exemplo, estudos relativos a análises de sentimento em fóruns de discussão de MOOCs (Massive Online Open Courses - Cursos On-line Abertos e Massivos) visando descobrir antecipadamente prováveis situações de abandono (WEN, YANG e ROSÉ, 2014 apud GIL et al., 2015); assim, além de realizar essa descoberta, pode-se tentar considerar as emoções dos alunos para realizar adaptações no ambiente - usando-se, por exemplo, agentes pedagógicos animados (NUNES e JAQUES, 2014) - com o objetivo de mantê-los engajados nesses cursos, reduzindo, consequentemente, as taxas de evasão.

O reconhecimento de emoções do usuário geralmente requer dispositivos específicos, uma vez que podem ser reconhecidas pelas seguintes fontes: (i) voz (prosódia) ou diálogo; (ii) comportamento observável, isto é, as ações do usuário na interface do sistema (por exemplo, opções escolhidas e velocidade de digitação); (iii) expressões faciais; e (iv) sinais fisiológicos (batimentos cardíacos, eletromiograma - tensão muscular, condutividade da pele, respiração) (JAQUES E NUNES, 2012).

Um modelo afetivo que se enquadra como uma abordagem multidimensional de emoção é o PAD - Pleasure, Arousal e Dominance (MEHRABIAN, 1996). A dimensão Pleasure trata a valência da emoção sentida (variando de "agradável" a "desagradável"), a dimensão Arousal trata o grau de excitação (variando entre "excitado" e "relaxado") e a dimensão Dominance trata o grau de controle do usuário (variando de "dominante" até "submisso"). Ressalta-se que, além do PAD, há outros modelos para representar emoções, como o modelo OCC ${ }^{1}$ (ORTONY, CLORE e COLLINS, 1988), por meio do qual é explicada a origem de 22 tipos de emoções, sendo descritos os processos cognitivos que ativam cada um deles.

Para definir os valores de cada dimensão do modelo PAD, Bradley e Lang (1994) apresentaram o Self-Assessment Manikin (SAM), um instrumento orientado a figuras. Broekens e Brinkman (2009) apresentaram o AffectButton, uma ferramenta para a detecção do estado afetivo do usuário a partir de feedback explícito, construída de formar similar ao SAM, na qual o usuário pode informar dinamicamente o seu estado. Na ferramenta (Figura 2), é exibida uma face que muda de expressão a partir do movimento do mouse e, a partir disso, são medidos os valores para cada dimensão do modelo PAD.

Considerando, por exemplo, que, ao tentar solucionar determinados problemas, o ato de programar computadores gera diferentes emoções ao programador aprendiz, este cenário é um possível caso para aplicar o conceito de emoções visando à melhoria da educação na área da computação. A identificação das emoções poderia ser útil em diversas pesquisas a fim de

\footnotetext{
${ }^{1}$ De acordo com Longhi (2011), o modelo OCC (ORTONY, CLORE e COLLINS, 1988) - provavelmente o mais utilizado para avaliar/sintetizar emoções de usuários em sistemas computacionais - pressupõe que as percepções emocionais de um agente são valoradas a partir de seus objetivos, padrões e preferências. Neste modelo, considera-se que uma emoção é derivada a partir da sua avaliação sob três dimensões: (i) consequência dos eventos, (ii) ação dos agentes e (iii) aspectos dos objetos, permitindo, além de inferir uma emoção, verificar a experiência subjetiva e a valência emocional.
} 
sugerir meios facilitadores para o desenvolvimento de alguns programas, com base em escolhas/caminhos seguidos anteriormente por outros usuários/programadores que, ao final da realização de tarefas similares, indicaram uma emoção indicativa de sucesso, a exemplo de alegria.

Um exemplo de estudo considerando emoções é descrito por Pereira (2012), no qual se utilizou o contexto afetivo do usuário para propor um Sistema de Recomendação que faz uso das emoções do usuário para selecionar e sugerir itens durante o processo de recomendação: o Affective-Recommender, que utiliza o modelo PAD e o instrumento SAM (a partir do AffectButton).

Figura 2 - AffectButton (de esquerda para a direita, de cima para baixo, faces geradas para os estados: Feliz, Com medo, Surpreso, Triste, Bravo, Relaxado, Contente, Frustrado).

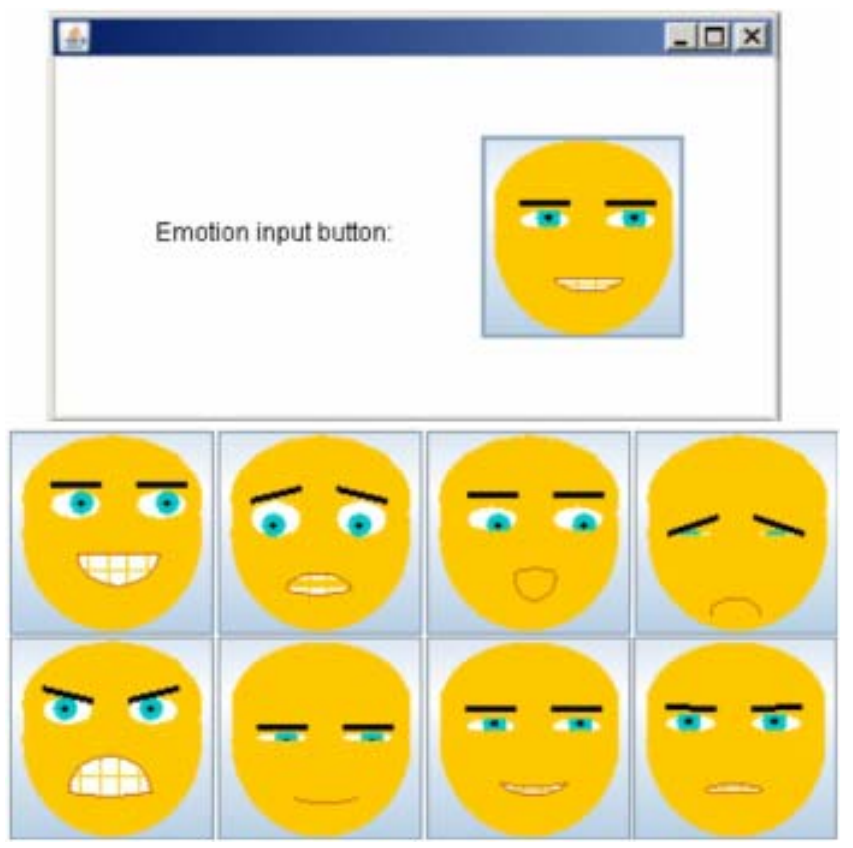

Fonte: Broekens e Brinkman (2009), p. 3.

A recomendação de itens no Affective-Recommender é realizada baseada em usuários com preferências similares. A preferência de um usuário para um item é vista como sua reação estado afetivo detectado após o contato - ao item. Os resultados indicaram que os alunos conseguiram informar seus estados afetivos, e que houve uma mudança neste estado baseado no item acessado (PEREI RA, 2012).

O uso de um Sistema de Recomendação baseado em Emoções, como o AffectiveRecommender, é um exemplo de iniciativa que poderia ser adotada em cursos na modalidade a distância, e até mesmo em cursos presenciais, contanto que utilizassem o Moodle como ambiente virtual de apoio ao processo de ensino e aprendizagem. 
Uma vez que emoções são mais breves/momentâneas, variando em curto período de tempo (diferentemente dos Estilos de Aprendizagem e da Personalidade de um indivíduo), não são apresentados nesta seção diagramas (como feito nas Seções 3 e 5 - Figuras 1 e 3 ) com perfis de alunos de computação relativos a emoções.

\section{Personalidade}

A Personalidade pode ser definida como padrão consistente do comportamento originado internamente no indivíduo (BURGER, 2000), embora não haja um consenso na Psicologia para sua definição.

Nunes (2012) afirma que a Teoria do Traço (ALLPORT e ALLPORT, 1921) tem sido a mais comumente aplicada para representação de personalidade, por ser mais facilmente mensurável de forma explícita em humanos por meio de computadores.

O modelo dos Cinco Grandes Fatores (CGF) da personalidade, ou Big Five (JOHN e SRIVASTAVA, 1999), é uma versão moderna da Teoria do Traço. Os CGF, em português, podem ser definidos como: Extroversão, Socialização (ou Amabilidade), Realização (ou Conscientização), Neuroticismo (ou Instabilidade Emocional) e Abertura (ou Abertura à mudança). No Quadro 3, são apresentados adjetivos característicos do Big Five.

Quadro 3 - Adjetivos característicos do Big Five.

\begin{tabular}{|c|c|c|c|c|c|}
\hline & Extroversão & Socialização & Realização & Neuroticismo & Abertura \\
\hline & Ativo & Altruísta & Confiável & Ansioso & Artístico \\
& Aventureiro & Amigável & Consciente & Apreensivo & Curioso \\
& Barulhento & Carinhoso & Eficiente & Emotivo & Engenhoso \\
& Energético & Confiante & Minucioso & Instável & Esperto \\
& Entusiástico & Cooperativo & Organizado & Nervoso & Imaginativo \\
& Exibido & Gentil & Prático & Preocupado & Inteligente \\
& Sociável & Sensível & Preciso & Temeroso & Original \\
& Tagarela & Simpático & Responsável & Tenso & Sofisticado \\
\hline \multirow{4}{*}{ Polo do rótulo o oposto } & Acanhado & Antipático & Desatento & Calmo & Comum \\
& Introvertido & Brigão & Descuidado & Contido & Simples \\
& Quieto & Bruto & Desorganizado & Estável & Superficial \\
& Reservado & Crítico & Distraído & Indiferente & Tolo \\
& Silencioso & Frio & Imprudente & Sereno & Trivial \\
& Tímido & Insensível & Irresponsável & Tranquilo & Vulgar \\
\hline
\end{tabular}

Fonte: Nunes (2012), p. 119.

Para extrair informações relativas à personalidade humana, é comum utilizar inventários de personalidade. Dentre os validados, há o NEO-IPIP (Neo-International Personality Item Pool), que permite medir as 5 dimensões do Big Five, incluindo mais 6 facetas para cada dimensão, propiciando maior precisão na representação da personalidade. O NEO-IPIP é um inventário com 300 itens que possuem afirmativas, aos quais o respondente atribui um valor de concordância do quanto essas afirmativas o representam em uma escala tipo Likert de cinco pontos. Uma alternativa ao NEO-IPIP é o inventário TIPI (Ten I tem Personality Inventory), com apenas 10 itens (NUNES, 2012). 
Um exemplo de iniciativa em Informática na Educação utilizando Personalidade é a ferramenta Group Recommender (NUNES et al., 2010), que possibilita a criação de equipes de trabalho eficientes coordenadas por tutores, considerando as características do tutor e a similaridade de Traços de Personalidade de seus alunos. Para utilização do sistema, é necessário que os usuários/alunos respondam ao Personality Inventory PV1.0 (NUNES, MORAES e REINERT, 2010) - uma interface para o NEO-IPIP e TIPI, com versão em português -, do qual se extrai o modelo de personalidade e, por meio deste, são gerados os dados necessários para a criação das equipes (NUNES, 2012).

Segundo Gosling (2008), a melhor forma de obtenção dos Traços de Personalidade dos usuários seria a utilização de abordagens que não exigissem esforço cognitivo (como, por exemplo, padrão de digitação) se comparado aos tradicionais inventários de personalidade. Neste contexto, há pesquisas, tais como a de Bachrach et al. (2012), a de Markovikj et al. (2013) e a de Schwartz et al. (2013), que descrevem que Traços de Personalidade possuem correlação com padrões de uso da rede social Facebook (https://www.facebook.com/), podendo facilitar a detecção de personalidade com a mineração dos dados dos usuários dessa rede social.

Na Figura 3, são ilustrados em gráficos alguns perfis de alunos de computação, a partir de seus Traços de Personalidade, extraídos via ferramenta Five Labs (FIVE, 2015), que se baseia no estudo de Schwartz et al. (2013).

Figura 3 - Exemplos de perfis de alunos de computação, com Traços de Personalidade distintos, em diferentes níveis de ensino: (a) técnico; (b) graduação; (c) mestrado; (d) doutorado.

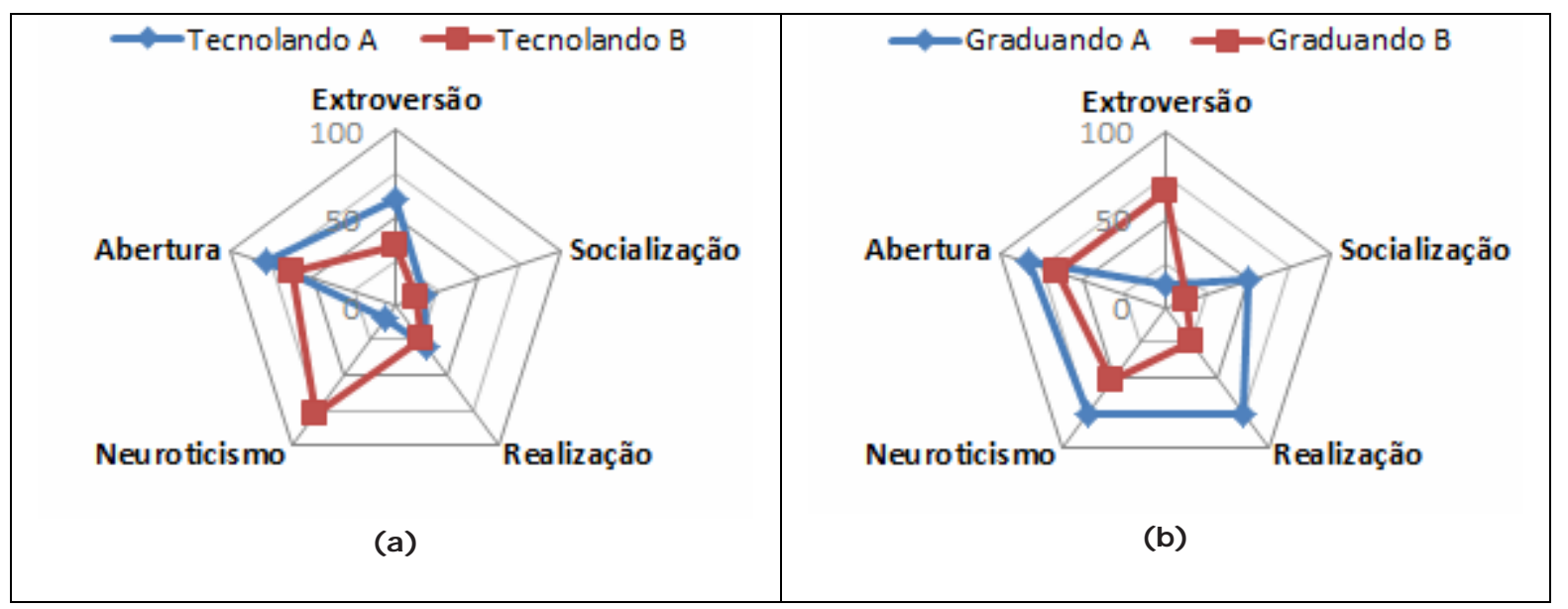




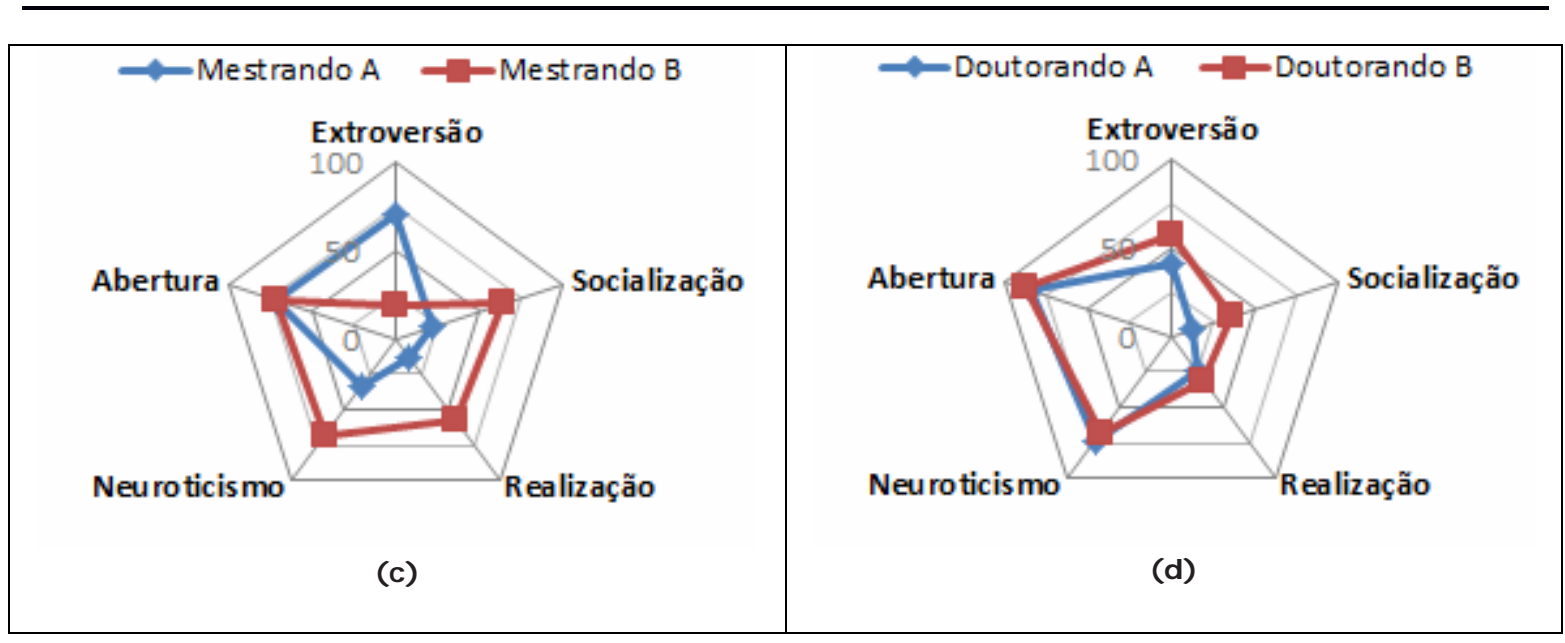

Fonte: elaborada pelo autor.

Similarmente à comparação relativa a estilos de aprendizagem (Figura 1), nota-se, a partir da Figura 3, diferenças entre perfis de alunos em um curso técnico, na graduação, no mestrado e no doutorado. Com base nos gráficos 3(a), 3(b), 3(c) e 3(d), é possível observar, entre os alunos de computação, a não homogeneidade de perfil relativa aos Traços de Personalidade, independente no nível de ensino. Destaca-se, por exemplo, o alto nível de Neuroticismo no Tecnolando B em relação ao Tecnolando A, e o baixo nível de Extroversão do Graduando A em relação ao Graduando B.

Os dados apresentados na Figura 3 foram obtidos com base na participação de 81 alunos de computação (38 alunos em nível técnicos, 23 alunos de graduação e 20 - mestrandos ou doutorandos), os quais informaram, via formulário on-line, os resultados da ferramenta Five Labs. A partir das respostas, foi definido, para caracterizar cada aluno, um vetor com 5 elementos (dados numéricos entre 0 a 100) representando o valor percentual de cada um dos traços do Big Five (Extroversão, Socialização, Realização, Neuroticismo e Abertura). Uma vez que cada aluno foi representado por um vetor, foi utilizada a Medida de Similaridade dos Cossenos para identificar os pares de alunos menos similares (assim como no estudo relativo a Estilos de Aprendizagem - Seção 3), ilustrando-se na Figura 3 seus perfis.

Assim como comentado na Seção 3 (sobre a Figura 1, relativa a estilos de aprendizagem), ressalta-se que os gráficos da Figura 3 não são apresentados com o objetivo de delinear um perfil de Personalidade dos alunos de tais cursos. O objetivo consiste em destacar, a partir desses exemplos, a diferença na personalidade dos alunos em uma mesma área/nível de ensino.

Considerando que pessoas com personalidades similares podem ter preferências similares, a utilização de sistemas de recomendação educacionais, utilizando a estratégia de recomendação baseada em personalidade (NUNES e HU, 2012), consiste em outro interessante cenário possível para aplicar aspectos psicológicos em Informática na Educação.

Voltando-se à área da computação para exemplificar o uso de personalidade em I nformática na Educação, é interessante citar que, em uma recente revisão sistemática de pesquisas sobre 
personalidade em engenharia de software (CRUZ, DA SILVA e CAPRETZ, 2015), foi percebido que pesquisas relacionadas a programação em pares, educação, eficácia da equipe, alocação de processo de software, características de personalidade dos engenheiros de software e performance individual concentraram mais de $88 \%$ dos estudos selecionados. Acredita-se que, além da computação, várias outras áreas podem se beneficiar de estudos relativos à formação de equipes, além de aspectos relacionados à execução de tarefas específicas nas variadas áreas do conhecimento humano.

\title{
6 Considerações Finais
}

A partir dos fundamentos apresentados, é possível notar que há várias formas de expandir o uso de aspectos psicológicos em Informática na Educação, a exemplo de pesquisas no âmbito da personalização de ambientes virtuais de aprendizagem, que tende a impactar positivamente no desempenho individual dos aprendizes. A personalização é uma característica importante em sistemas para EAD devido às diferentes experiências dos alunos adultos, foco principal desse tipo de modalidade de ensino (KERKIRI, MANITSARIS e MAVRIDOU, 2007).

Como afirmado anteriormente, os aspectos psicológicos - tais como Emoções e Traços de Personalidade - influenciam processos de tomada de decisão. Uma vez que tomar decisões é algo cotidiano para os indivíduos, tais conceitos apresentam-se potencialmente úteis para a melhoria do processo de ensino e aprendizagem. Compete, neste ponto, expor esta consideração de J aques e seus colaboradores:

\begin{abstract}
Ainda, embora existam importantes trabalhos que apontem a importância de considerar as emoções na aprendizagem, não existem teorias compreensíveis e validadas que identifiquem quais emoções são mais importantes para aprendizagem e como especificamente estas interferem na aprendizagem. Dessa forma, devido também a sua natureza multidisciplinar, essa área se mostra ao mesmo tempo um campo de pesquisa desafiador e promissor. (J AQUES et al., 2012, s/p.)
\end{abstract}

É notável, portanto, que a Computação Afetiva consiste em um campo propício para a realização de investigações científicas que possibilitem ainda mais o uso das TDIC para a melhoria da educação. Aliado a isso, considerar os Estilos de Aprendizagem é essencial para dar suporte às diferenças diretamente relacionadas ao modo particular de aprender dos alunos.

O desenvolvimento de iniciativas nessa linha de pesquisa apresenta-se como algo desafiador, uma vez que a forma de extrair esses aspectos psicológicos e unir esses conceitos, para proporcionar um desempenho maior no processo de ensino e aprendizagem, requer reflexões e implementações não triviais.

A relevância de trabalhos nessa temática volta-se ao aspecto da preocupação com as características humanas dos estudantes. A educação é uma área cujos aspectos psicológicos influenciam diretamente devido à psicologia também se preocupar em como um indivíduo adquire conhecimento. 
Destaca-se também que, além dos conceitos em foco nesta pesquisa, há outros fatores afetivos que têm influência na aprendizagem, como os estados de ânimo (SCHERER, 2000) conceito brevemente comentado na Seção 4.

Finalizando com comentários relativos à computação (área escolhida neste artigo para exemplificar a aplicação dos conceitos expostos nas Seções 3 a 5), é possível dizer, por exemplo, que a aprendizagem de programação é um problema extremamente relevante, mas é diferente de aprender engenharia de software (que envolve, dentre outros aspectos, o trabalho em equipes, gerenciamento de recursos e pessoas etc.); além disso, aprender computação teórica é mais similar com a aprendizagem de matemática e mais diferente de, por exemplo, aprender sobre redes, sistemas operacionais e arquitetura de computadores. Ou seja, dentro de uma mesma área, é possível encontrar uma variedade grande de contextos que poderiam se beneficiar dos conceitos de estilos de aprendizagem, emoções e/ou personalidade.

\section{Agradecimento}

À Coordenação de Aperfeiçoamento de Pessoal de Nível Superior (CAPES), pelo apoio financeiro, e aos alunos que responderam aos questionários.

\section{Referências}

AGUIAR, J. J. B.; FECHINE, J. M.; COSTA, E. Estilos Cognitivos e Estilos de Aprendizagem em Informática na Educação: um mapeamento sistemático focado no SBIE, WIE e RBIE. In: Anais do 25o Simpósio Brasileiro de Informática na Educação, Dourados, p. 441-450, 2014.

ALLPORT, F. H.; ALLPORT, G. W. Personality Traits: Their Classification And Measurement. Journal Of Abnormal And Social Psychology, 16, p. 6-40, 1921.

BACHRACH, Y.; KOSINSKI, M.; GRAEPEL, T.; KOHLI, P.; STILLWELL, D. Personality and patterns of Facebook usage. In: Proceedings of the 3rd Annual ACM Web Science Conference (WebSci '12), ACM, New York, NY, USA, p. 24-32, 2012.

BRADLEY, M. M.; LANG, P. J. Measuring emotion: the self-assessment manikin and the semantic differential. Journal of Behavior Therapy \& Experimental Psychiatry, v.25, n.1, p. 49-59, 1994.

BROEKENS, J.; BRINKMAN, W.-P. AffectButton: Towards a Standard for Dynamic Affective User Feedback. In: Affective Computing and Intelligent Interaction and Workshops. p. 1-8, 2009.

BURGER, J. M. Personality. Wadsworth, fifth edition, 2000.

COFFIELD, F.; MOSELEY, D.; HALL, E.; ECCLESTONE, K. Should we be using learning styles? What research has to say to practice. London, Learning and Skills Research Centre, Learning and Skills Development Agency. 2004. Disponível em:

<http://www. itslifejimbutnotasweknowit.org.uk/files/LSRC_LearningStyles.pdf>. Acesso em: 09 jun. 2016.

COSTA, E.; AGUIAR, J.; MAGALHÃES, J. Sistemas de Recomendação de Recursos Educacionais: conceitos, técnicas e aplicações. In: II Congresso Brasileiro de Informática na Educação - J ornada de Atualização em Informática na Educação (JAIE), p. 57-78, 2013.

CRUZ, S.; DA SILVA, F. Q. B.; CAPRETZ, L. F. Forty years of research on personality in software engineering: A mapping study. Computers in Human Behavior, v. 46, p. 94-113, 2015. 
DORÇA, F. A.; LIMA, L. V.; FERNANDES, M. A.; LOPES, C. R. Comparing strategies for modeling students learning styles through reinforcement learning in adaptive and intelligent educational systems: An experimental analysis. Expert Systems with Applications, v. 40, n. 6, p. 2092-2101, 2013.

FAQUIN, G. S.; FALCI, M. L. F.; ARAÚJO, M. A. P. Uma Metodologia de Avaliação da Relação entre Perfis de Personalidade e Desempenho Acadêmico em Alunos de Sistemas de Informação. In: Proceedings of the XII Brazilian Symposium on Information Systems, Florianópolis, p. 285-292, 2016.

FELDER, R. M.; SILVERMAN, L. K. Learning and Teaching Styles in Engineering Education. Journal of Engineering Education, v. 78, n. 7, p. 674-68, 1988.

FELDER, R.; SOLOMAN, B. A. Index of Learning Styles (ILS). 1999. Disponível em: <http://www4.ncsu.edu/unity/lockers/users/f/felder/public/ILSpage.html>. Acesso em: 09 jun. 2016.

FELDER, R.; SPURLIN, J. Applications, Reliability, and Validity of the Index of Learning Styles. International Journal of Engineering Education, v. 21, p. 103-112, 2005.

FIVE. Five Labs - See the personality behind your posts. Product of Five.com. 2015. Disponível em: <http://labs.five.com/>. Acesso em: 24 abr. 2015.

GIL, R.; VIRGILI-GOMÁ, J.; GARCÍA, R.; MASON, C. Emotions ontology for collaborative modelling and learning of emotional responses. Computers in Human Behavior, v. 51, p. 610-617, 2015.

GOSLING, S. Psiu, Dê Uma Espiadinha: O Que As Suas Coisas Dizem Sobre Você; Tradução Marcio De Paula S. Hack. Rio De Janeiro: Elsevier. 2008.

GRAF, S.; KINSHUK; LIU, T-C. I dentifying Learning Styles in Learning Management Systems by Using Indications from Students' Behaviour. In: ICALT '08 - Eighth IEEE International Conference on Advanced Learning Technologies, p. 482-486, 2008.

JAQUES, P. A.; NUNES, M. A. S. N. Ambientes Inteligentes de Aprendizagem que inferem, expressam e possuem emoções e personalidade. In: Isotani, S.; Campos, F. C. A. (Org.). Jornada de Atualização em Informática na Educação - JAIE 2012. 1 ed. Porto Alegre: SBC, v. 1, p. 32-71, 2012.

J AQUES, P. A.; NUNES, M. A.; ISOTANI, S.; BITTENCOURT, I. Computação Afetiva aplicada à Educação: Dotando Sistemas Tutores Inteligentes de Habilidades Sociais. In: Anais do CSBC 2012 - DesafIE, Curitiba, 2012.

JOHN, O. P.; SRIVASTAVA, S. The Big Five Trait taxonomy: History, measurement, and theoretical perspectives. In: PERVIN, L. A.; JOHN, O. P. (Org.), Handbook of Personality: Theory and research, v. 2, p. 102-138. Guilford Press, New York. 1999.

KERKIRI, T.; MANITSARIS, A.; MAVRIDOU, A. Reputation Metadata for Recommending Personalized eLearning Resources. In: International Workshop on Semantic Media Adaptation and Personalization, Los Alamitos, CA, USA, v.0, p. 110-115, 2007.

KOMULAINEN, E.; MESKANEN, K.; LIPSANEN, J.; LAHTI, J.; JYLHÄ, P.; MELARTIN, T.; WICHERS, M.; ISOMETSÄ, E.; EKELUND, J. The Effect of Personality on Daily Life Emotional Processes. PLoS ONE, v. 9, n. 10 , p. 1-9, 2014.

LI, M.; ARMSTRONG, S. J. The relationship between Kolb's experiential learning styles and Big Five personality traits in international managers. Personality and Individual Differences, v. 86, p. 422-426, 2015.

LITZI NGER, T. A.; LEE, S. H.; WISE, J. C.; FELDER, R. M. A Psychometric Study of the Index of Learning Styles. Journal of Engineering Education, 96(4), p. 309-319, 2007.

LONGHI, M. T. Mapeamento de aspectos afetivos em um ambiente virtual de aprendizagem. Tese (Doutorado) - Instituto de Informática, Universidade Federal do Rio Grande do Sul, Porto Alegre, 2011. Disponível em: <http://hdl.handle.net/10183/39578>. Acesso em: 30 jul. 2016. 
MARKOVIKJ, D.; GIEVSKA, S.; KOSINSKI, M.; STILLWELL, D. Mining Facebook Data for Predictive Personality Modeling. Proc of Workshop on Computational Personality Recognition, AAAI Press, Melon Park, CA. 2013.

MEHRABIAN, A. Pleasure-arousal-dominance: A general framework for describing and measuring individual differences in Temperament. Current Psychology, v. 14, p. 261-292, 1996.

NUNES, M. A. S. N. Computação Afetiva personalizando interfaces, interações e recomendações de produtos, serviços e pessoas em Ambientes computacionais. In: NUNES, M. A. S. N.; OLIVEIRA, A. A.; ORDONEZ, E. D. M. (Org.). Projetos e Pesquisas em Ciência da Computação no DCOMP/PROCC/UFS: São Cristóvão, p. 115-151, 2012.

NUNES, M. A. S. N.; BeZERRA, J. S.; REINERT, D.; MORAES, D.; PEREIRA, E.; PEREIRA, A. J. S. Computação Afetiva e sua influência na personalização de Ambientes Educacionais: Gerando equipes compatíveis para uso em AVAs na EaD. In: Glaucio J osé Couri Machado. (Org.). Educação e Ciberespaço: Estudos, Propostas e Desafios. Aracaju: Virtus Editora, v. 1, p. 308-347, 2010.

NUNES, M. A. S. N.; HU, R. Personality-based recommender systems: an overview. In: Proceedings of the sixth ACM conference on Recommender systems (RecSys '12). ACM, New York, NY, USA, p. 5-6, 2012.

NUNES, M. A. S. N.; MORAES, D.; REINERT, D. Personality Inventory - Pv 1.0 (Portuguese Version). Registrado no Inpi. 2010. Disponível em: <http://personalityresearch.ufs.br/pt/produtos/softwares/personality-inventory/versao-10>.

NUNES, T. M.; JAQUES, P. A. Utilizando Agentes Pedagógicos Animados como uma abordagem não restritiva ao Gaming The System. Revista Brasileira de Informática na Educação, v. 22, n. 1, p. 147-163, 2014.

ORTONY, A.; CLORE, G.; COLLINS, A. The cognitive structure of emotions. Cambridge, UK. 1988.

ÖZYURT, Ö.; ÖZYURT, H. Learning style based individualized adaptive e-learning environments: Content analysis of the articles published from 2005 to 2014. Computers in Human Behavior, v. 52, p. 349-358, 2015.

PEREIRA, A. Affective-Recommender: Um Sistema de Recomendação Sensível ao Estado Afetivo do Usuário. Dissertação de Mestrado, Universidade Federal de Santa Maria - Centro de Tecnologia, Santa Maria, Rio Grande do Sul, Brasil. 2012.

POPESCU, E.; TRIGANO, P.; BADICA, C. Adaptive Educational Hypermedia Systems: A Focus on Learning Styles. In: EUROCON 2007 - The International Conference on "Computer as a Tool”, p. 2473-2478, 2007.

REIS, R. C. D.; RODRIGUEZ, C. L.; LYRA, K. T.; JAQUES, P. A.; BITTENCOURT, I. I.; ISOTANI, S. Estado da Arte sobre Afetividade na Formação de Grupos em Ambientes Colaborativos de Aprendizagem. Revista Brasileira de Informática na Educação, v. 23, n. 3, p. 113-130, 2015.

SAMSUDIN, Z.; CH'NG, L. The Learning Styles And Learning Emotions Of Adult Learner In E-Learning Environment. International Educational Technology Conference (IECT), Istanbul - Turkey, 2015.

SCHERER, K. Psychological models of emotion. In: BOROD, J. (Ed.). The neuropsychology of emotion. Oxford/New York: Oxford University Press. p. 137-162, 2000.

SCHWARTZ, H. A.; EICHSTAEDT, J. C.; KERN, M. L.; DZIURZYNSKI, L.; RAMONES, S. M.; AGRAWAL, M.; SHAH, A.; KOSINSKI, M.; STILLWELL, D.; SELIGMAN, M. E. P.; UNGAR, L. H. Personality, Gender, and Age in the Language of Social Media: The Open-Vocabulary Approach. PLoS ONE, 8(9), e73791, 2013.

SILVA, D. M.; LEAL, E. A.; PEREIRA, J. M.; OLIVEIRA NETO, J. D. Estilos de Aprendizagem na Educação a Distância: Uma Investigação em Cursos de Especialização. In: EnANPAD 2013, Rio de Janeiro. 2013.

TRUONG, H. M. Integrating learning styles and adaptive e-learning system: Current developments, problems and opportunities. Computers in Human Behavior, v. 55, p. 1185-1193, 2016. 
VALASKI, J.; MALUCELLI, A.; REINEHR, S. Revisão dos Modelos de Estilos de Aprendizagem Aplicados à Adaptação e Personalização dos Materiais de Aprendizagem. In: XXII Simpósio Brasileiro de Informática na Educação - SBIE, p. 844-847, 2011.

ZYWNO, M. S. A Contribution to Validation of Score Meaning for Felder-Soloman's Index of Learning Styles". In: Proceedings of the 2003 American Society for Engineering Education Annual Conference \& Exposition. Nashville, Tennessee. 2003. Disponível em:

<http://www4.ncsu.edu/unity/lockers/users/f/felder/public/ILSdir/Zywno_Validation_Study.pdf>. Acesso em: 09 jun. 2016.

Recebido em junho de 2016

Aprovado para publicação em agosto de 2017

\section{Janderson Jason Barbosa Aguiar}

Programa de Pós-Graduação em Ciência da Computação - Universidade Federal de Campina Grande UFCG, Brasil, janderson@copin.ufcg.edu.br 